\title{
Accessory foramen in the human mandible: anatomical and clinical considerations
}

\author{
Farihah Haji Suhaimi · Srijit Das
}

Received: 10 May 2012/ Accepted: 22 May 2012/Published online: 7 June 2012

(C) Japanese Association of Anatomists 2012

We read with interest the published article 'Anatomical classification of accessory foramina in human mandibles of adults, infants, and fetuses' by Przystańska and Bruska (2012). The authors performed a meticulous study of the accessory foramen in the mandible. We wish to share few scientific facts related to the published topic.

It is always better to visualize an accessory foramen in a radiograph. The best possible means is to insert a wire and visualize the continuity of the canal. An accessory foramen is meant for passage of the neurovascular structures. It would be better to interpret the concerned neurovascular structures according to the position of the accessory foramen. A past study cut the mandible in the midline and measured the dimensions of the canal under dissecting microscope (Liang et al. 2007). Even though the accessory foramina were discussed, no speculation was made about the blood vessels or nerves traversing it. The authors mentioned in their discussion that the accessory foramen was connected to the mandibular foramen. Radiographic investigations may provide exact evidence continuity of the accessory foramina with the mandibular canal but it is quite difficult to believe such in the absence of radiographic studies. The accessory foramen has also been reported to have continuity with the incisive canal (von Arx et al. 2011). If there is continuity of the accessory foramen with the mandibular canal, one wonders if the mental nerve emerges out through this foramen. This raises serious issues for successful nerve blocks. Previous studies have described consideration of additional locoregional anaesthesia in the case of failed mandibular block anesthesia (von Arx et al. 2011).

F. H. Suhaimi · S. Das $(\bowtie)$

Department of Anatomy, Faculty of Medicine,

Universiti Kebangsaan Malaysia, Jalan Raja Muda Abd Aziz,

50300 Kuala Lumpur, Malaysia

e-mail: drsrijit@gmail.com
In the second paragraph of the results section, under the subheading Adult mandibles, it was mentioned that four different types of foramina were found but only three were mentioned in pointwise form. We wonder if this really refers to types of foramina or do the authors mean different positions of the foramina.

Contrary to the present findings, past research studies depict the accessory foramen varying according to age and race (Kaufman et al. 2000). Interestingly, an earlier study speculated that eruption of wisdom teeth may require additional neurovascular nourishment (von Arx et al. 2011). The present study should have discussed the eventualities of eruption of wisdom teeth or even additional dental spurs. Calculation of the mean distance of this canal with respect to a bony point may have been more beneficial to maxillofacial surgeons. These may be needed for planning graft implants.

We congratulate the authors for their valiant effort, and thank the editors for publishing an interesting topic.

Conflict of interest The authors have no conflict of interest.

\section{References}

Kaufman E, Serman NJ, Wang PD (2000) Bilateral mandibular accessory foramina and canals: a case report and review of the literature. Dentomaxillofac Radiol 29:170-175

Liang X, Jacobs R, Lambrichts I, Vandewalle G (2007) Lingual foramina on the mandibular midline revisited: a macroanatomical study. Clin Anat 20:246-251

Przystańska A, Bruska M (2012) Anatomical classification of accessory foramina in human mandibles of adults, infants, and fetuses. Anat Sci Int. doi:10.1007/s12565-012-0136-Z

von Arx T, Matter D, Buser D, Bornstein MM (2011) Evaluation of location and dimensions of lingual foramina using limited conebeam computed tomography. J Oral Maxillofac Surg 69:27772785 